\title{
L'éducation pilier du développement économique et humain au Maroc
}

Hassan Marhnine ${ }^{\text {a* }}$ \& Chafika Zarhouti ${ }^{\mathrm{a}}$

${ }^{a}$ Centre Régional des Métiers de L'Education et de la Formation de la Région de Tanger-Tétouan-Al Hoceima, Morocco

*radimarhn@hotmail.com 


\section{L'éducation pilier du développement économique et humain au Maroc}

\section{Résumé}

Cette recherche s'inscrit dans une perspective de la mise en évidence des retombés de l'éducation et la formation sur la qualification et la promotion des compétences professionnelles des ressources humaines. Elle permet en outre de mettre en exergue la corrélation dialectique et substantielle entre l'expansion du capital humain et le développement économique, social et culturel des différentes nations. Au demeurant, il est communément admis chez les différents acteurs politiques ou économiques que l'enseignement et la formation des cadres sont d'une importance majeure dans les progrès réalisés par les nations dans les divers secteurs et même un vecteur incontournable de leur renaissance. Il en ressort que les gouvernements de divers pays ne ménagent guère d'efforts à investir massivement dans ce secteur de l'éducation sans toutefois se soucier des budgets colossaux que cela nécessite et des retombés immédiats qu'ils devraient impérativement engendrer sur les tissus économique et social.de la société du moment qu'ils ont la conviction que les bonnes performances du système éducatif se répercutent inéluctablement à court, à moyen ou à long terme sur les divers secteurs productifs et ce par les richesses qu'elles sont susceptibles de générer. Cette recherche est structurée selon deux volets, l'un théorique et un autre pratique et ce comme suit : le volet théorique met l'accent sur les facteurs clés qui interviennent dans la production des richesses et qui par conséquent actionnent le développement économique et social, en l'occurrence, le capital humain et le capital financier. Le volet pratique est dédié à une étude statistique relative à la corrélation entre le capital humain et la croissance économique. La recherche est close par certaines suggestions inhérentes au thème débattu dans cet article.

Mots-clés : éducation, développement économique, développement humain, capital humain. 


\title{
Education, a pillar of economic and human development in Morocco
}

\begin{abstract}
This research is part of a perspective of highlighting the impact of education and training on the qualification and promotion of professional human resources skills. It also helps to highlight the dialectical and substantial correlation between the expansion of human capital and the economic, social and cultural development of different nations. Moreover, it is commonly accepted among the various political or economic actors that education and training is of major importance in the progress made by nations in the various sectors and even an essential vector of their rebirth. It shows that the governments of various countries are sparing no effort in investing heavily in this education sector. They do not care at all about the colossal budgets that this requires, and the immediate repercussions that they should imperatively generate on the economic and social aspects, as long as they are convinced that the good performance of the education system inevitably has repercussions in the short term, in the medium or long term in the various productive sectors, through the wealth they are likely to generate. This research is structured in two parts, one theoretical and another practical, as follows: the theoretical part emphasizes the key factors that intervene in the production of wealth and which consequently drive economic and social development, in this case, human capital and financial capital. The practical part is dedicated to a statistical study relating to the correlation between human capital and economic growth. The research is closed by certain suggestions inherent to the topic discussed in this article.
\end{abstract}

Keywords: education, economic development, human development, human capital.

\section{Introduction}

En quête du chemin du progrès et de la relance économique et sociale tant convoités, les gouvernements de divers pays ne cessent de prôner des politiques spécifiques en matière de l'éducation et de la formation, d'entreprendre des reformes appropriées, de procéder à des réajustements et de changer même de stratégies inhérentes à l'éducation. 
Dans cette perspective, on peut signaler à titre indicatif que le Maroc avait initié, depuis son indépendance, plusieurs projets de réformes structurelles relatives au système de l'éducation et de la formation en vue d'en améliorer la qualité et en tirer les résultats escomptés. Cependant le projet phare que le Maroc adoptait et qui s'inscrivait dans cette fin remonte à l'aube de l'année 2009 et qui était connu sous le nom du plan d'urgence lequel avait mobilisé un investissement colossal dans le capital humain et l'implication de plusieurs partenaires. Nonobstant l'engagement et la grande volonté du gouvernement en place, plusieurs facteurs, y compris la mauvaise gouvernance, le manque de suivi et la passivité ont interféré pour entraver finalement le plan en question d'arriver à maturité surtout après l'arrivée d'une nouvelle équipe gouvernementale. Celle-ci s'est engagée activement à se lancer dans la recherche d'un plan alternatif en invitant des partenaires crédibles et sérieux à y prendre part, en l'occurrence le conseil supérieur de l'éducation, de la formation et de la recherche scientifique (CSEFRS). Ce conseil a œuvré inlassablement à élaborer ce nouveau plan connu sous le nom de « la Vision Stratégique 2015-2030 » qui servira comme une feuille de route pour les réformes à entreprendre au profit de l'enseignement et formation afin que ce secteur joue le rôle éminent qui lui échoit celui de l'épanouissement du capital humain soit le levier du développement économique, social et culturel. Dans la perspective d'atteindre cet objectif crucial et donc de gagner le pari, les intervenants dans la vision stratégique ont bel et bien souligné la nécessité d'investir généreusement dans les ressources humaines, soit le capital humain.

En sciences économiques, il est bien connu que les facteurs clés dans la production des richesses sont naturellement le capital financier et le travail. Ces deux facteurs influencent mathématiquement le produit intérieur brut (PIB) d'un pays. Rappelons, à ce propos, que le PIB en nominal est un agrégat macroéconomique qui 
mesure la production des richesses d'un pays et il est défini comme étant la somme des valeurs ajoutées réalisées à l'intérieur d'un pays par l'ensemble des agents économiques : entreprises publiques ou privées, ménages et administrations publiques, indépendamment de la nationalité des entreprises qui s'y trouvent, auxquelles on doit ajouter la TVA et les droits de douane, et ce au titre d'un exercice donné soit :

$$
P I B_{\text {Nominal }}=V A_{\text {E.publiques }}+V A_{\text {E.privées }}+T V A+\text { Droits de Douane } .
$$

Quant à la croissance économique, celle-ci se mesure grâce à son taux égal à la différence entre les PIB de deux années consécutives $n$ et $n-1$ quotientée par l'année $n-1$; soit

$$
t=\frac{P I B(n)-P I B(n-1)}{\text { année } n-1} \times 100
$$

A titre indicatif, la banque africaine de développement BAD et le fond monétaire international FMI mettent en avant que le PIB du Maroc au titre de l'exercice 2017 est estimé à 121.4 milliards USD ce qui lui vaut le soixantième rang à l'échelle mondiale et juste le statut de la sixième puissance africaine après avoir perdu le cinquième rang au profit de la république du Soudan. A titre de comparaison avec des pays voisins, il s'agit d'une place honorable pour un pays qui ne dispose guère de ressources naturelles notables telles que le pétrole, le gaz ou encore les métaux précieux. En revanche, s'il l'on compare la situation économique du Maroc avec certains pays ayant une taille semblable à la sienne comme la Corée du Sud, son économie parait faible devant l'économie sud-coréenne dont le PIB est estimé au titre de l'exercice 2017 à 1 trillion 500 milliards USD soit la onzième puissance économique mondiale avec un indice de développement humain IDH très fort de l'ordre de 0.901 surclassant ainsi de grandes puissances classiques comme la Russie et l'Espagne. A 
l'aube de la sixième décennie du siècle dernier, la Corée du Sud était plongée dans la précarité et la misère et sa situation économique et sociale était comparable à celle du Maroc à cette époque. Et d'ajouter que ce pays, comme ce fut le cas du Maroc, ne disposait plus de ressources naturelles notables sur lesquelles il pouvait compter pour bâtir son économie, d'autant plus que son relief et sa géographie ne sont pas favorables à un développement économique et se trouve souvent contraint d'importer la quasitotalité des produits agricoles et les produits énergétiques dont il a besoin pour dynamiser son économie. Partant de tout ce constat, une question émerge de toute évidence, à savoir, quel est le secret du miracle économique de ce pays surnommé dragon asiatique ? Les spécialistes en la matière conviennent systématiquement et à l'unanimité que seules les ressources humaines hautement qualifiées en matière de compétences professionnelles soit un capital humain à haute valeur ajoutée expliquent le boum économique et la montée en puissance de ce pays pourtant nain en termes de superficie géographique de l'ordre de $100000 \mathrm{Km}^{2}$ soit environ $1 / 7$ de la superficie du Maroc. Il convient d'ajouter en outre que l'investissement massif dans le capital humain justifie également et sans nul doute le succès économique retentissant d'autres pays comme la Malaisie, Singapour, Taiwan ou encore le Japon.

\section{Etude Théorique}

Dans cette partie, on procède à une étude théorique en revenant sur les différentes réflexions, les perceptions, les pensées et les théories relatives au capital humain et les divers investissements dans ce type de capital en liaison avec l'éducation et la formation.

\section{Capital humain}

Le concept de capital humain a été développé avant tout en 1961 par l'économiste 
américain Theodore Schultz (1961) spécialiste en économie du développement et prix Nobel d'économie en 1979 qu'il partage avec Arthur Lewis. Il l'exprime en termes suivants : «Alors qu'il apparait évident que les individus acquièrent des savoir-faire et des savoirs utiles, il n'est pas si évident que ces savoir-faire et savoirs constituent une forme de capital et que ce capital soit pour une part substantielle le produit d'un investissement délibéré ».

Ce concept a été reconsidéré et surtout caractérisé par l'économiste américain Gary Stanley Becker en 1964 et lui a valu le Prix Nobel de sciences économiques en 1992.

Dans son célèbre ouvrage «Human Capital », Gary Stanley Becker, définit le capital humain comme étant "l'ensemble des capacités productives qu'un individu acquiert par accumulation de connaissances générales ou spécifiques, de savoir-faire, etc." Becker met en évidence qu'il y a bien un investissement des ménages dans leur formation : plus précisément, il y a un arbitrage individuel entre les bénéfices attendus des années d'éducation et les coûts qu'elles impliquent. Ces coûts sont de deux ordres : les coûts directs, liés au financement de la formation, et les coûts d'opportunité, provenant du fait que les années de formation sont autant d'années non travaillées et donc non rémunérées pour l'individu. Les différences internationales dans la production et le financement de l'éducation agissent sur ces coûts et bénéfices et entraînent des attitudes différentes en termes d'effort des individus et d'organisation des systèmes éducatifs (sélectivité, importance du financement public...).

Selon la définition de l'Organisation de Coopération et de Développement Economique (OCDE), le capital humain recouvre « l'ensemble des connaissances, qualifications, compétences et caractéristiques individuelles qui facilitent la création du bien-être personnel, social et économique. » «Le capital humain constitue un bien 
The Journal of Quality in Education (JoQiE) Vol.10, N¹6, November 2020

immatériel qui peut faire progresser ou soutenir la productivité, l'innovation et l'employabilité ».

Une autre perception du capital humain a été mise au point par Fuente et Ciccone. En effet, selon eux, le capital humain d'un individu est défini surtout par les connaissances et compétences que ce dernier maîtrise. Ces connaissances et compétences se sont accumulées tout au long de la scolarité, au cours des diverses formations suivies et à l'occasion des expériences vécues (Fuente et Ciccone, 2002). On peut en distinguer trois composantes essentielles (Fuente et Ciccone, 2002) : les compétences générales (alphabétisation, calcul de base, capacités d'apprentissage), les compétences spécifiques liées aux technologies ou aux processus de production (programmation informatique, entretien et réparation des pièces mécaniques) et les compétences techniques et scientifiques (maîtrise de masses organisées de connaissances et de techniques analytiques spécifiques).

Les compétences acquises par un individu au cours de sa formation contribuent à distinguer son capital humain et à en faire une ressource rare. Comme l'explique Marshall (1894), cette rareté est rémunérée sous la forme d'une « quasi-rente », compensant l'individu pour son effort de formation et visant à donner aux futurs offreurs de travail l'incitation nécessaire pour acquérir et renouveler les compétences spécifiques et valorisables dans la production.

L'acquisition de ces compétences modifie également le comportement d'offre de travail des individus, qui aspirent alors à une rémunération plus élevée. Les individus plus qualifiés sont prêts à expérimenter une période d'inactivité plus longue en attendant de trouver une offre d'emploi conforme à leurs aspirations. Les différences de stratégies de recherche d'emploi mettent en exergue l'importance de l'appariement entre l'entreprise et le futur employé. La sélection d'un candidat à l'embauche présente 
un risque pour l'entreprise, surtout si celle-ci doit investir dans des formations spécifiques. Il s'agit donc pour les employeurs de trouver l'employé ou le cadre le plus productif pour le poste à pourvoir.

Selon l'économiste américain Andrew Michael Spence (1973), prix Nobel d'économie en 2001 avec Joseph E. Stiglitz et George Akerlof pour ses analyses du marché en situation d'asymétrie d'information, l'investissement dans le capital humain serait surtout pour une personne un moyen de signaler ses capacités aux entreprises, bien plus que de les accroître. En effet, le coût de l'éducation est censé être plus faible pour les individus dotés de certaines capacités. L'acquisition de connaissances leur est plus facile (moindre coût en termes d'effort, de concentration), ils s'attendent à recevoir des salaires futurs élevés qui compenseront les salaires perdus aujourd'hui (arbitrage intertemporel qui réduit l'importance des coûts d'opportunité). Aussi, seules les personnes les plus productives trouveront rentable de réaliser cet investissement. Cette sélection permet dès lors de signaler les capacités des personnes.

Cette vision permet en quelque sorte d'expliquer la perte de valeur des diplômes et autres reconnaissances institutionnelles dans les sociétés qui ont connu une massification scolaire. Cela dit, il n'est pas question de retirer aux formations leur rôle d'acquisition de connaissances et de savoir-faire ; d'ailleurs, les entreprises comme les individus investissent dans la formation même après l'embauche.

Au niveau macroéconomique, le capital humain apparaît ainsi comme un facteur de croissance. Mankiw, Romer et Weil (1992) prouvent que les différences de capital humain entre différents pays du globe permettent d'expliquer une grande partie de leurs écarts en termes de croissance économique et de PIB. Si leur évaluation approximative du capital humain d'un pays en fonction de la proportion de ses ressources humaines ayant suivi des études secondaires reste critiquée, ils n'ont pas moins ouvert la voie aux 
nouvelles théories de la croissance endogène. Ces dernières sont cruciales car elles permettent de comprendre pourquoi les niveaux de richesses des pays ne sont pas amenés à converger : du fait de l'importance de l'innovation et des complémentarités entre les compétences individuelles, des différences de stock et d'accumulation de capital humain génèrent des différences de taux de croissance qui perdurent.

\section{Typologie}

Si le capital humain se définit, au niveau d'une entreprise, par les connaissances maîtrisées par un individu, force est de constater qu'il recouvre des catégories représentant des enjeux différents pour les entreprises en termes de contrôle.

Il est en effet possible de dresser une typologie du capital humain qui distingue les catégories suivantes : capital humain général, capital humain spécifique à l'entreprise, capital humain spécifique à une tâche (Gibbons et Waldman, 2004 ; Hatch et Dyer, 2004).

Le capital humain général correspond à des connaissances qui ne sont ni spécifiques à une entreprise, ni à une fonction ou à une tâche singulière. Il s'agit de connaissances et de compétences génériques (discernement, capacités d'analyse, intelligence des situations) essentiellement accumulées par les expériences professionnelles et l'éducation.

Le capital humain spécifique à la tâche se constitue essentiellement au moyen de formations professionnelles et d'expériences professionnelles. Il correspond à des compétences qui sont spécifiques à un poste de travail comme assistant de direction, auditeur financier ou Risk-manager.

Quant au capital humain spécifique à l'entreprise, il correspond à des compétences et des connaissances maîtrisées par un salarié basées sur un corpus de connaissances et de connaissances collectives (capital organisationnel) spécifique à une 
entreprise donnée. Le capital humain spécifique à l'entreprise octroie à un collaborateur des capacités directement liées à des besoins spécifiques à une entreprise en particulier. Aussi, lorsqu'un individu doté d'un capital humain spécifique à la firme quitte celle au sein de laquelle il l'a essentiellement développé pour une autre société, une grande partie de ce capital humain ne sera pas utilisé (les attentes et les besoins de la nouvelle entreprise sont différents de la précédente) (Gibbons et Waldman, 2004). C'est pourquoi, ce type de capital humain, parce qu'il se déprécie dès que son détenteur (le salarié) quitte la firme au sein de laquelle il a accumulé le capital, s'avère moins intéressant pour d'autres entreprises. C'est la raison pour laquelle il est plus aisé à contrôler pour l'entreprise au sein de laquelle il s'est développé.

En revanche, le capital humain général et le capital humain spécifique à la tâche, sont facilement « expropriables » dans la mesure où ils ont presque autant de valeur pour la firme au sein de laquelle les collaborateurs «louent » ce type de capital que pour d'autres entreprises.

Quelles que soient la catégorie, le capital humain correspond à des connaissances susceptibles d'améliorer la productivité de la firme. Cette relation de causalité (capital humain ? accroissement de la productivité ? accroissement du revenu d'emploi) est un des postulats de la théorie néoclassique du capital humain. Reste à fonder cette proposition, ce qui, comme on va le voir, soulève plusieurs difficultés épistémologiques

Une analyse minutieuse des ces différentes pensées permettent de mettre en exergue le constat selon lequel elles convergent toutes vers la contribution large du capital humain comme capital immatériel à la production économique tout comme le capital financier et qu'il est absolument nécessaire de conjuguer ces deux facteurs si l'on veut effectivement booster la croissance économique et sociale. En effet, les 
compétences professionnelles, les savoirs, les savoir-faire et les expériences tels qu'ils sont évoqués dans les diverses définitions du capital humain se répercutent sur le facteur travail quantifiable par le biais du nombre d'heures et la production de la richesse qui en résulte. Et d'ajouter, comme l'ont signalé Guillard et Roussel (2010), le marché du travail fonctionnerait grâce à une convention de capital humain entendue comme convention d'évaluation de la compétence des personnes dont l'objet est de fonder la hiérarchie des salaires, sans laquelle employeurs et employés ne pourraient faire face à l'incertitude caractérisant la relation d'emploi. Cette convention de capital humain imposerait l'utilisation de la notion de capital humain comme évidente dans la gestion de la relation d'emploi et par voie de conséquence dans la gestion des ressources humaines.

\section{Etude statistique}

\section{Méthodologie}

Cette recherche se veut être aussi plus exhaustive et plus crédible si bien qu'on a envisagé de consulter les différentes opinions de diverses franges de la société. Par conséquent, un questionnaire a été confectionné à cette fin et distribué dans les villes de Tanger, Rabat et Meknès où on a pu bénéficier de la collaboration de certains partenaires que nous tenons à remercier chaleureusement.

La question globale de cette œuvre peut être formulée sommairement comme suit :

Quel est l'impact du capital humain sur le développement économique et social et quels sont les moyens mis en œuvre susceptibles d'épanouir ce capital humain? 
Tenant compte des objectifs escomptés de cette recherche, les questions sousjacentes de cette recherche peuvent se décliner comme suit :

(1) Que signifie le capital humain ? Masse populaire ou savoir-faire et compétences des individus ?

(2) Le capital humain contribue-t-il au développement économique et social ?

(3) L'enseignement contribue-t-il à la promotion du capital humain au Maroc ?

(4) Etes-vous satisfait(e) de l'état de santé de l'enseignement au Maroc ?

(5) L'enseignement, dans son état actuel, aurait-il des effets positifs tangibles sur l'économie marocaine?

(6) L'enseignement est-il en mesure de répondre aux besoins du marché d'emploi au Maroc?

(7) Quel est le moyen efficient pour promouvoir le capital humain au Maroc ?

(8) Quel est le moyen efficient pour Investir dans le capital humain C.H?

Le nombre global de personnes impliquées dans cette recherche était de l'ordre de 580 dont 436 avaient traité correctement le questionnaire et ont offert des données fiables. Le questionnaire contenait des questions fermées relatives aux questions préalablement évoquées. Les questions ouvertes ont été à leur tour introduites afin de permettre au public de s'exprimer avec une marge de liberté.

Les résultats sont présentés par le biais des tableaux explicatifs et représentés graphiquement afin de les rendre plus parlants et aisément interprétables. Ces résultats traduits graphiquement sont également affectés de certains brefs commentaires.

\section{Dépouillement des questionnaires et analyse qualitative}

Question 1. Que signifie pour vous le capital humain ? Masse populaire ou savoir-faire et compétences des individus ? 
Les résultats obtenus relativement à cette question étaient comme suit :

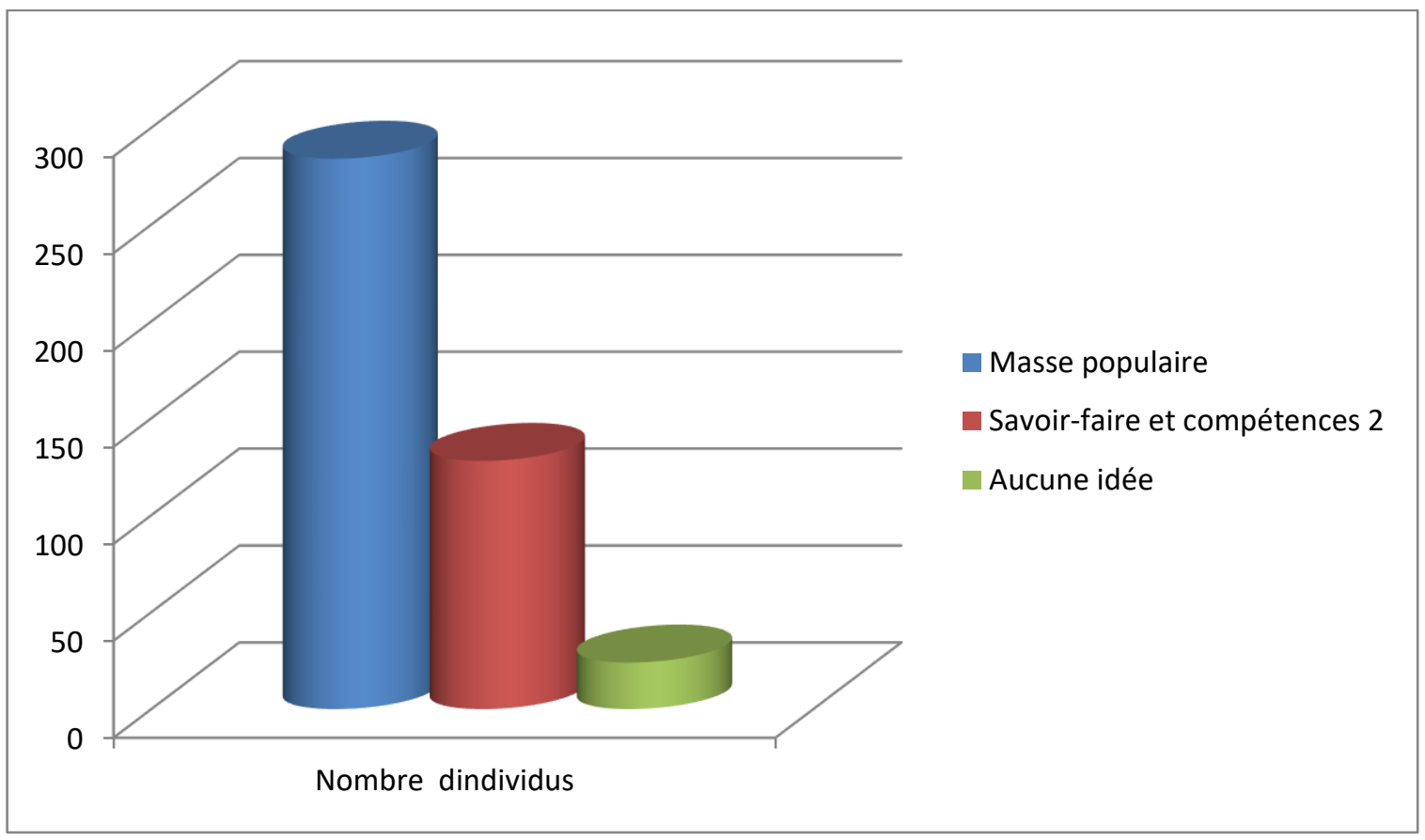

Graphe 1: Perception du capital humain

Ce graphe permet de conclure qu'une large majorité des marocains ignorent le concept du capital humain. Ce constat était d'ailleurs prévu. En effet, le concept est particulièrement connu chez les acteurs économiques et les spécialistes en économie. Cependant, en leur expliquant de quoi il s'agit, ils se sont impliqués dans notre action et ont répondu avec un sens de responsabilité inattendue.

Question 2. Le capital humain contribue-t-il au développement économique et social ?

Les résultats relatifs à cette question sont comme suit : 


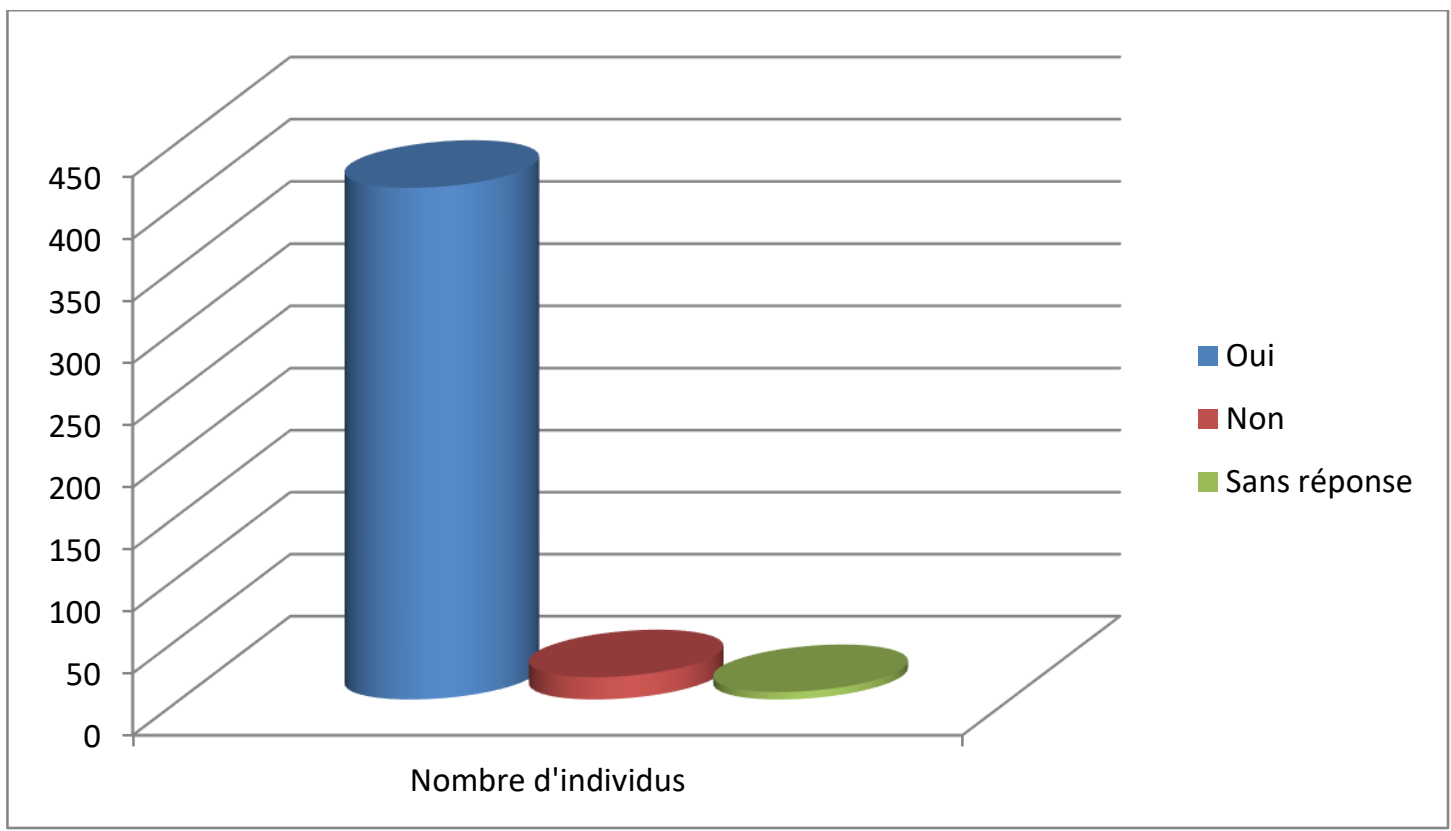

Graphe 2 : Contribution du capital humain au développement

Les chiffres sont très parlants dans ce cas de figure et prouvent sans nul doute que le capital humain contribue largement au développement économique et social.

Question 3. L'enseignement contribue-t-il à la promotion du capital humain au Maroc ?

Les réponses à cette question sont comme suit :

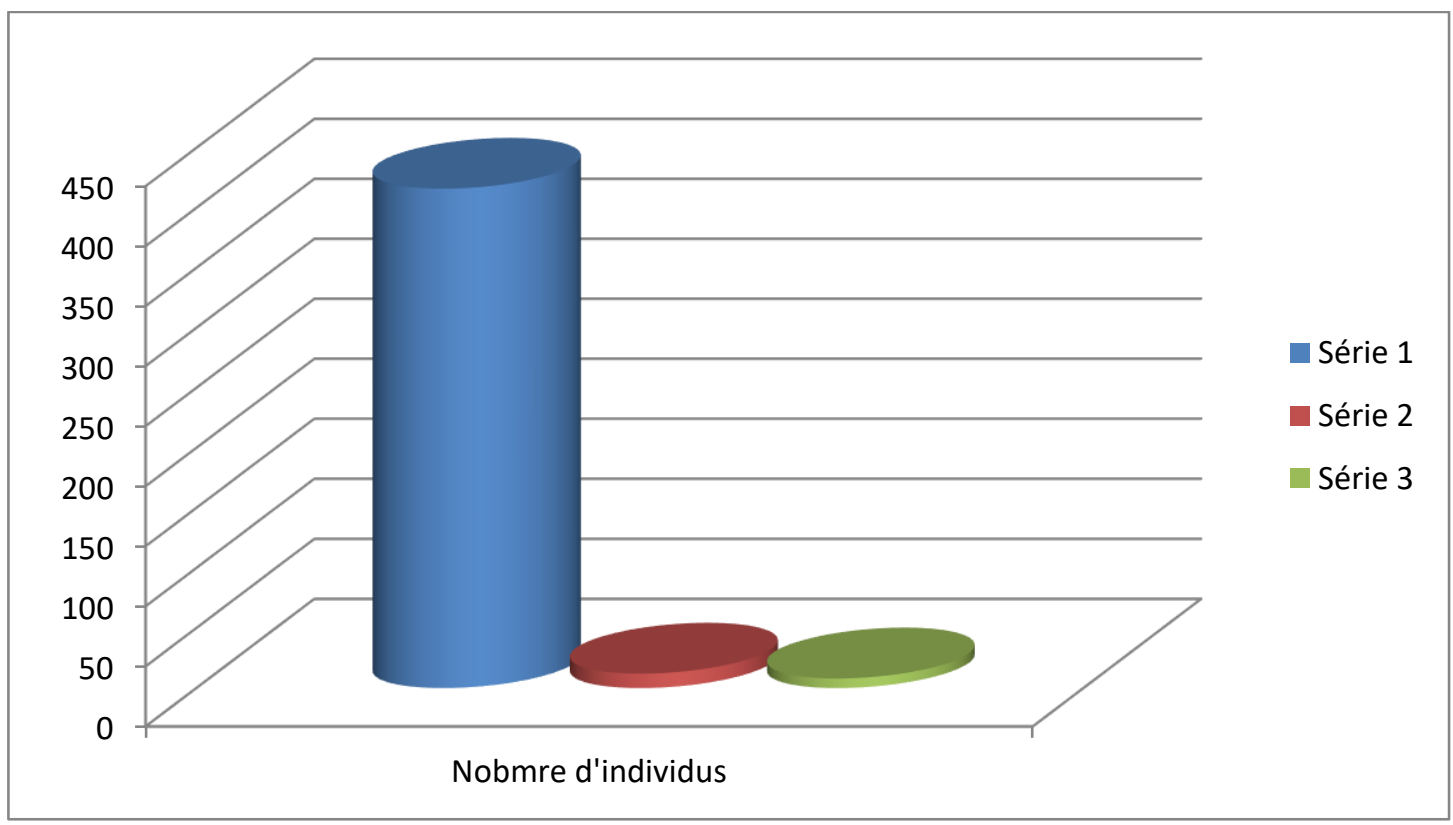

Graphe 3 : Contribution de l'enseignement au capital humain 
Les personnes interrogées sur ce point sont quasiment toutes unanimes quant à la contribution de l'enseignement à la promotion du capital humain au Maroc.

Question 4. Etes-vous satisfait (e) de l'état de santé de l'enseignement au Maroc ?

Les réponses relatives à cette question sont comme suit :

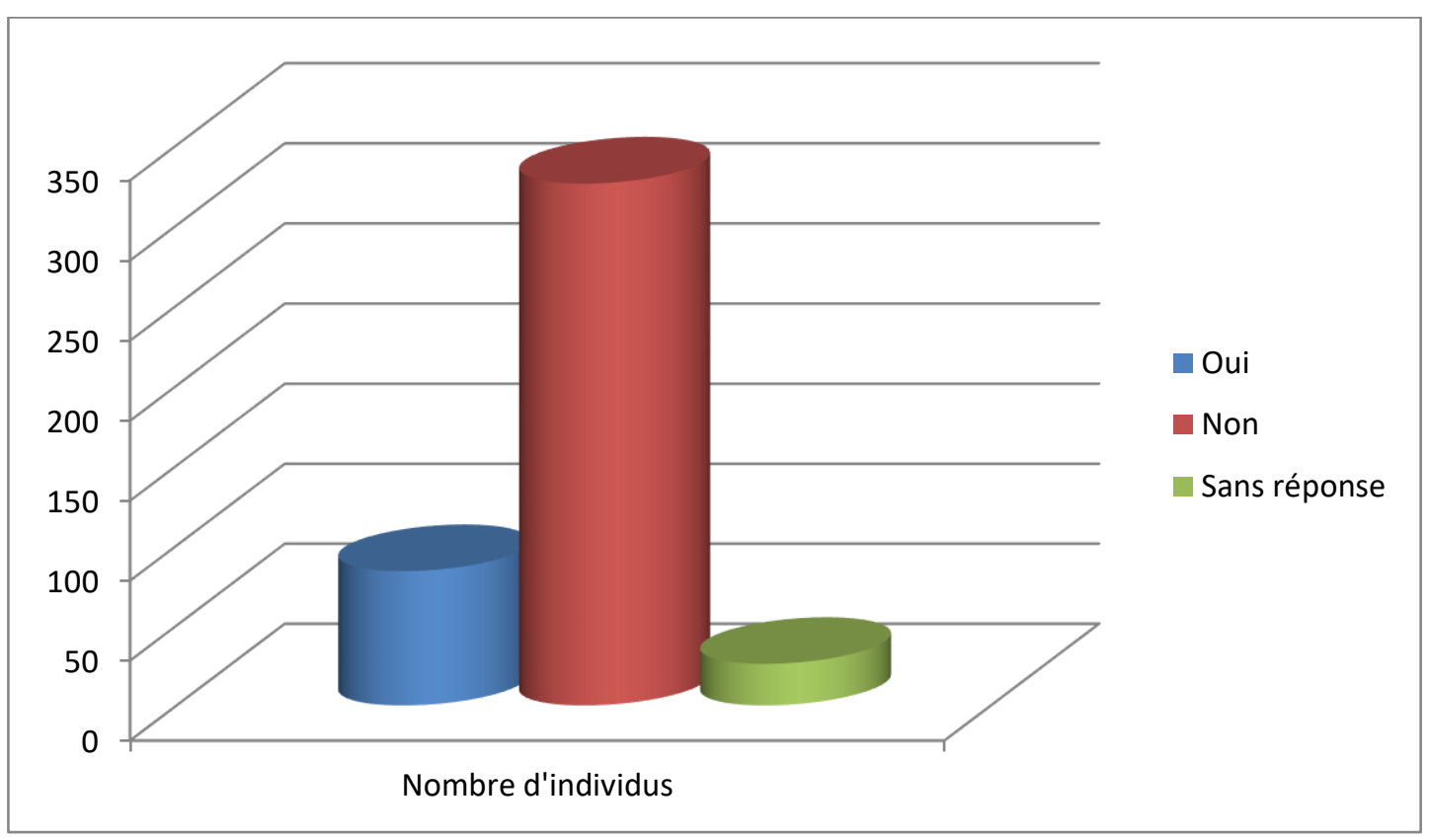

Graphe 4 : Taux de satisfaction sur l'état de santé de l'enseignement

Les réponses concernant cette question centrale avèrent explicitement que les marocains sont généralement insatisfaits du produit de leur enseignement, ce qui soulève beaucoup de questions à ce sujet.

Question 5. L'enseignement, dans son état actuel, aurait-il des effets positifs tangibles sur l'économie marocaine?

Les résultats relatifs à cette question sont comme suit : 


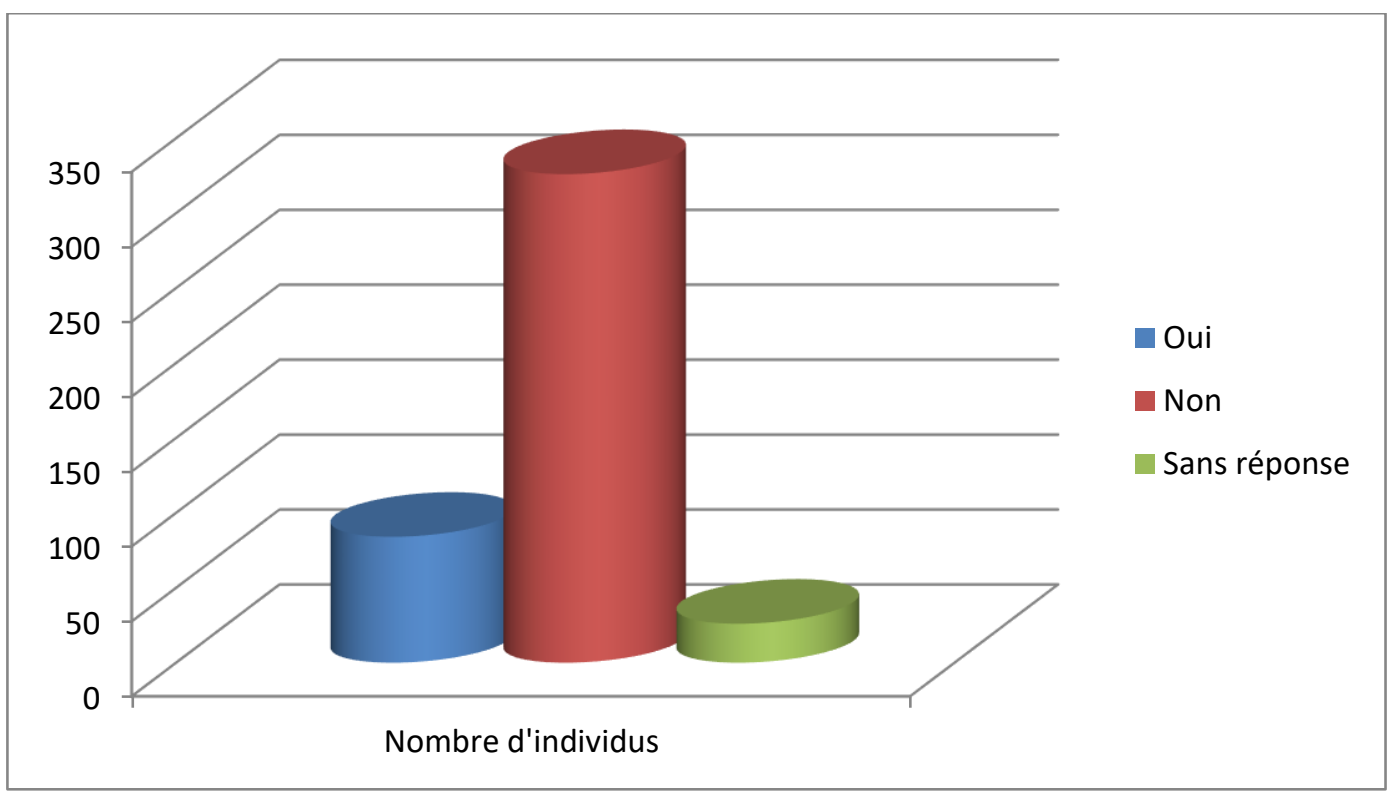

Graphe 5 : Effets actuels de l'enseignement sur l'économie

Ces résultats confirment l'attitude pessimiste des marocains à l'égard de l'enseignement au Maroc.

Question 6. L'enseignement est-il en mesure de répondre aux besoins du marché d'emploi au Maroc?

Les réponses relatives à cette question sont comme suit :

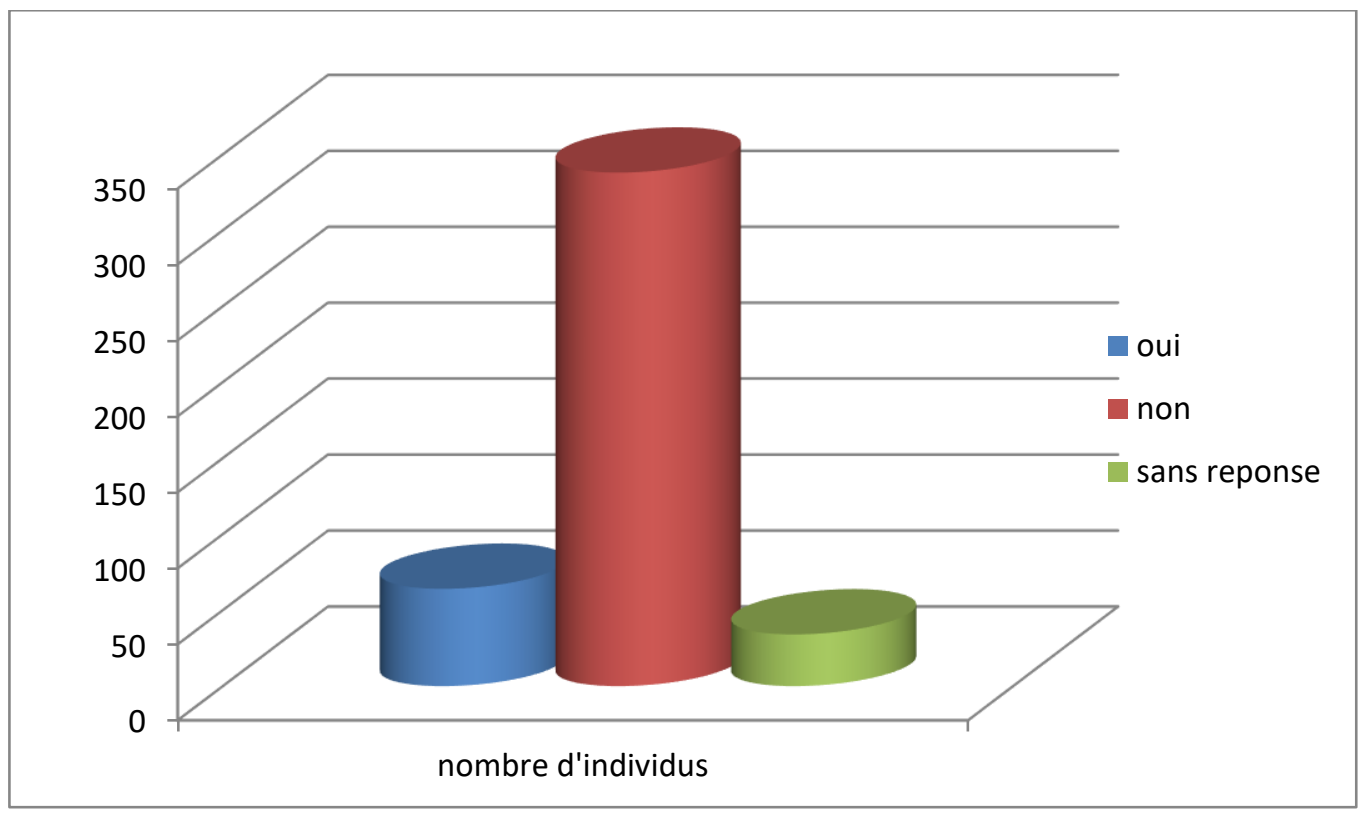

Graphe 6 : Adéquation formation-emploi 
Les réponses à cette question sont claires et laissent entendre que l'enseignement au Maroc n'est guère en adéquation avec les exigences du marché d'emploi et qu'il est en déphasage flagrant avec les compétences professionnelles exigées pour l'insertion dans la vie active. Cela explique d'ailleurs le taux très élevé du chômage chez les diplômés des universités et des grandes écoles.

Question 7. Quel est le moyen efficient pour élever la croissance économique au Maroc ? Investir dans le capital humain C.H, Donner des crédits, Emprunter chez le FMI ou la BM.

Les résultats relatifs à cette question sont :

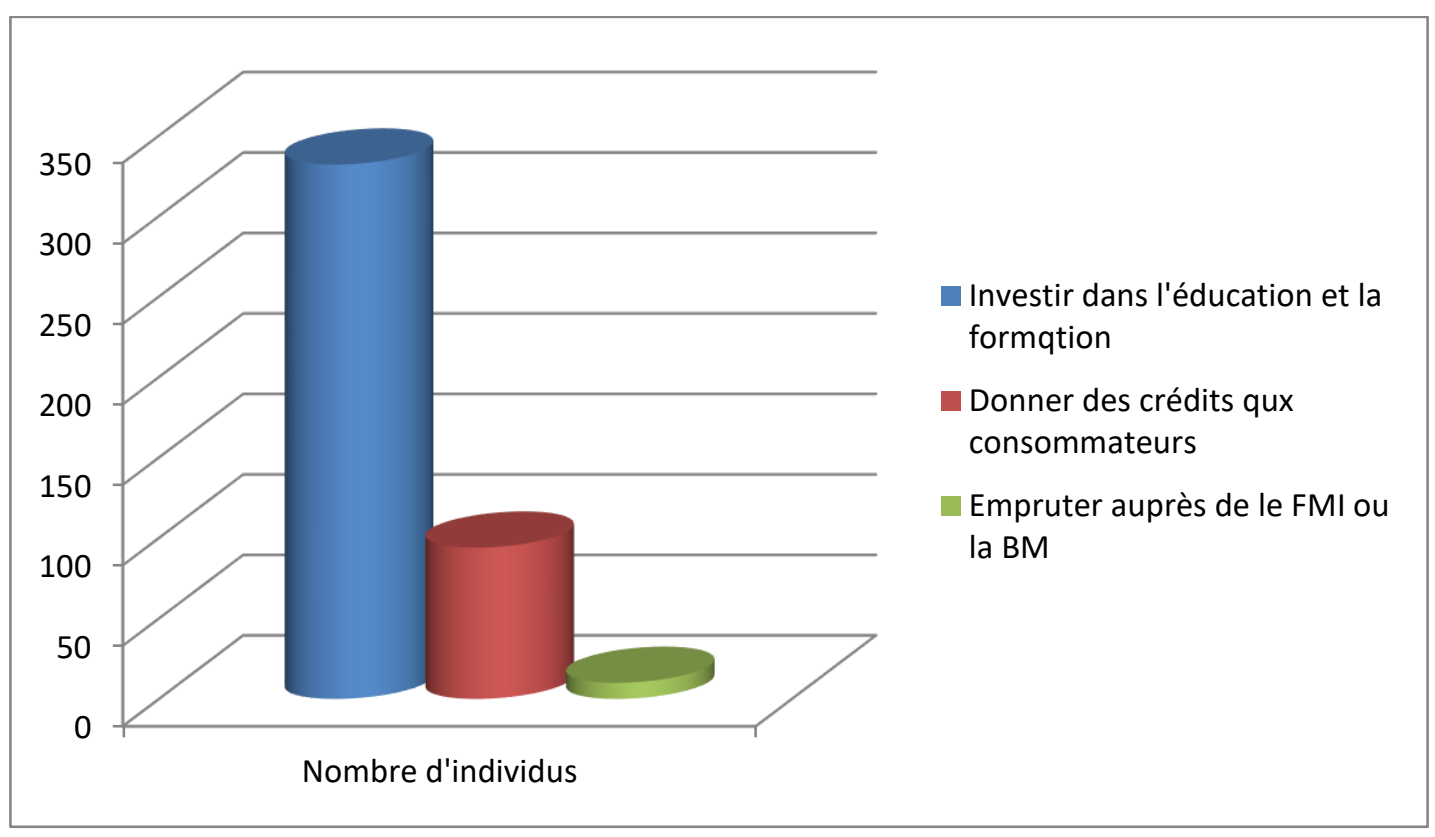

Graphe 7 : Importance de l'investissement dans le capital humain

Il découle de ces résultats que les marocains recommandent davantage l'investissement dans le capital humain et parient sur ce facteur pour stimuler la croissance économique et sont conscients du fait que les autres choix ne font qu'aggraver la situation à cause des dégâts qu'ils engendrent. 
Question 8. Quel est le moyen efficient pour investir dans le capital humain ?

Les résultats relatifs à cette question sont :

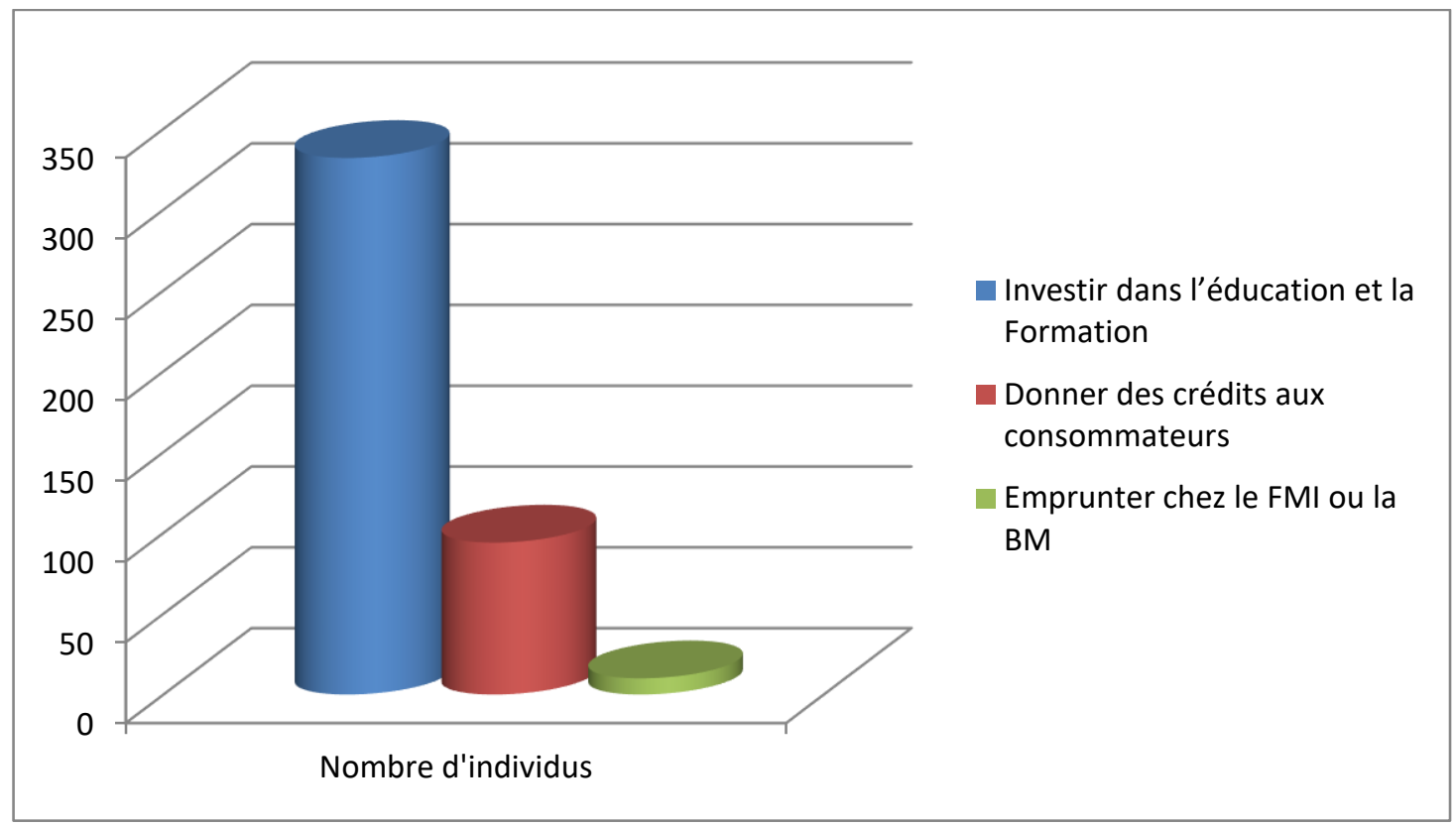

Graphe 8 : Le meilleur moyen d'investissement dans le capital humain

Les résultats décelés dans cette question laissent entendre que le meilleur moyen d'investir dans le capital humain consiste à investir dans l'éducation et la formation en insistant particulièrement sur le facteur qualité ce qui se répercuterait positivement sur la valeur ajoutée des ressources humaines détentrices de ce capital humain.

\section{Conclusion}

En vertu des diverses approches du capital humain évoquées dans l'étude théorique ainsi que les perceptions du public relatives à ce concept, il s'est avéré qu'il y a lieu d'une corrélation triangulaire liant trois facteurs clés dans cette étude, en l'occurrence, le capital humain, le développement économique et l'enseignement ou encore l'éducation et la formation. Il s'agit à vrai dire d'une corrélation de causalité entre ces trois paramètres dont chacun est conséquence de son prédécesseur est cause de son antécédent et que l'on peut schématiser par le diagramme suivant : 

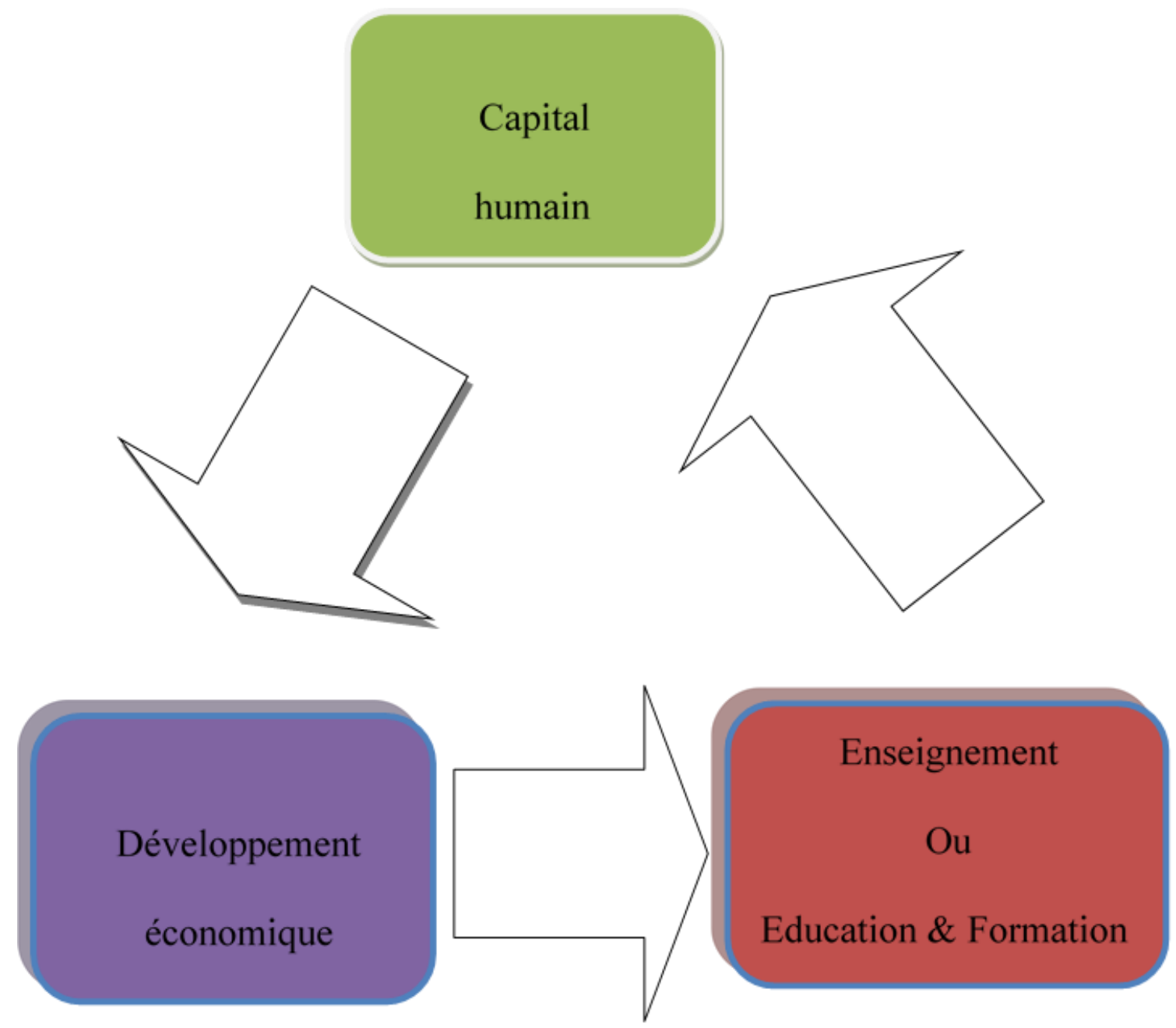

A l'instar des pays qui sont parvenus à réaliser une relance économique, le Maroc s'efforce de tracer son itinéraire vers le progrès et la croissance économique et sociale en se basant sur ses propres potentialités dont le capital humain. Cependant, conscient de sa contribution à son produit intérieur brut par sa haute valeur ajoutée et dans le but d'enrichir et rentabiliser ce capital humain, l'état marocain ne cesse de prôner des politiques qui feraient de l'éducation et la formation l'une des priorités majeures. En dépit de ces efforts et s'agissant d'un enjeu et d'un pari à gagner quelques soient les conjonctures, il est indéniablement recommandé au gouvernement marocain avec toutes ses composantes et en synergie avec le conseil supérieur de l'éducation, de la formation et de la recherche scientifique d'entreprendre un véritable débat national sur la problématique de l'éducation et la formation et qui implique les différents 
intervenants dans le système éducatif marocain, en l'occurrence les enseignants, les inspecteurs, les formateurs, les enseignants chercheurs, les cadres administratifs, les décideurs à l'administration centrale, la société civile représentée par les associations intéressées par l'éducation, les partis politiques et les centrales syndicales. Il appartient à toutes ces parties prenantes d'œuvrer inlassablement à enrichir davantage la Vision Stratégique 2015-2030 en matière d'innovations pédagogiques qui tiendraient compte du contexte et l'environnement socioculturels marocains. En effet, il ne s'agit plus d'importer une approche pédagogique de l'étranger sous prétexte qu'elle a permis d'avoir de bons résultats et de l'implémenter au système éducatif marocain car elle serait inévitablement vouée à l'échec à cause de la différence nette entre les environnements socioculturels respectifs. En outre, afin de gagner le pari, celui de disposer d'un capital humain riche et hautement qualifié, le Maroc devrait investir généreusement dans l'éducation et la formation en consacrant jusqu'à 7\% de son PIB à ce secteur vital et rompre avec le discours selon lequel l'enseignement est un secteur non productif et constitue un lourd fardeau pour la trésorerie de l'état.

Par ailleurs, pour saisir les opportunités offertes par le capital humain, fruit de l'investissement dans l'éducation et la formation, le Maroc est censé investir davantage dans des secteurs à haute valeur ajoutée tels que l'industrie automobile, l'aéronautique, l'électronique et technologie digitale et qui nécessitent un capital humain spécifique et hautement qualifié. En effet, il ne suffit plus de disposer d'un capital humain riche pour booster l'économie, il est impératif d'allier le capital financier et l'investir dans des projets impliquant le capital humain disponible. Dans ce sens, le gouvernement marocain est censé prôner une politique économique susceptible d'attirer plus d'investisseurs étrangers en leur procurant une infrastructure propice à l'investissement et en améliorant le climat des affaires par l'assainissement des secteurs qui l'influencent 
directement tel celui de la justice et la gouvernance. A ce titre, Doing Business classe le Maroc en 2017 au 68ème rang mondial en termes de facilité de faire des affaires surclassant ainsi l'Afrique du Sud. Il s'agit donc d'une place honorable si on la compare à celles d'autres pays de la région, mais il faudrait œuvrer davantage à renforcer sa position et la compétitivité de son économie qui reste vulnérable face aux conjonctures économiques, sociale et environnementale. Et de rappeler que l'économie marocaine demeure tributaire de la pluviométrie d'autant plus que le secteur de l'agriculture contribue à hauteur de $16.5 \%$ du PIB. Par ailleurs, lors du conseil du gouvernement tenu le 29/03/ 2018, le président du gouvernement a salué l'accès du Maroc au premier rang africain en 2017 en matière d'investissements étrangers directs en vertu du rapport publié ces derniers jours par le groupe international Quantum Global. Selon ce rapport, son attractivité en termes d'investissements se renforce et s'améliore en 2017. En effet, le Maroc a réussi à attirer environ 2.57 milliards USD ; soit une hausse de $12 \%$ en comparaison avec l'année 2016. En guise de conclusion, on estime que le meilleur moyen de stimuler la croissance économique consiste à promouvoir le capital humain et pour atteindre un tel objectif, il est impératif d'investir dans l'éducation et la formation en insistant particulièrement sur la qualité et l'adéquation de cette formation avec les exigences du marché de travail tout en suivant l'exemple coréen comme modèle de développement économique et social. Et d'ajouter que les intentions sincères et la bonne foi finissent par triompher.

\section{Références}

Becker G. (1964), Human Capital : A Theoretical and Empirical Analysis, with Special Reference to Education, University of Chicago Press.

De la Fuente A. et Ciccone A. (2002). Le capital humain dans une économie mondiale sur la connaissance. Rapport pour la Commission Européenne. 
Gibbons R. et Waldman M. (2004). « Task-specific human capital ». American Economic Review. Vol 94(2) : 203-207.

Guillard A. et Rossel J. (2010). Le capital humain en gestion des ressources humaines : éclairages sur le succès d'un concept. Revue Management \& Avenir. Vol. 31 (1) : 160-181.

Hatch N.W. et Dyer J.H. (2004). « Human capital and learning as a source of sustainable competitive advantage ». Strategic Management Journal. 25 :11551178.

Mankiw G., Romer D. et Weil D., «A Contribution to the Empirics of Economic Growth », Quarterly Journal of Economics.

Marshall (1894), Principles of Economics, London : Macmillan.

Schultz T.W. (1961). «Investment in human capital ». American Economic Review, n51, pp.1-17.

Spence A. M. (1973), « Job Market Signaling », Quarterly Journal of Economics.

L'investissement dans le capital humain, OCDE, 1998 ; Du bien-être des nations, le rôle du capital humain et social, OCDE, 2001. 